\title{
Fe-Vacancy-Ordered $\mathrm{Fe}_{4} \mathrm{Se}_{5}$ : The Insulating Parent Phase of FeSe Superconductor
}

\author{
Keng-Yu Yeh ${ }^{1,2,3}$, Yan-Ruei Chen ${ }^{1,2,3}$, Tung-Sheng Lo ${ }^{1}$, Phillip M. Wu ${ }^{1,4}$, Ming-Jye Wang ${ }^{1,5}$, \\ Kuei-Shu Chang-Liao ${ }^{3}$ and Maw-Kuen $\mathrm{Wu}^{1 *}$ \\ ${ }^{1}$ Institute of Physics, Academia Sinica, Taipei, Taiwan, ${ }^{2}$ Nano Science and Technology Program, Taiwan International Graduate \\ Program, Academia Sinica, Taipei, Taiwan, ${ }^{3}$ Department of Engineering and System Science, National Tsing Hua University, \\ Hsinchu, Taiwan, ${ }^{4}$ BitSmart LLC, San Mateo, CA, United States, ${ }^{5}$ nstitute of Astronomy and Astrophysics, Academia Sinica, \\ Taipei, Taiwan
}

We carried out a detailed study to investigate the existence of an insulating parent phase for FeSe superconductors. The insulating $\mathrm{Fe}_{4} \mathrm{Se}_{5}$ with $\sqrt{ } 5 \times \sqrt{ } 5 \mathrm{Fe}$-vacancy order shows a 3DMott variable-range-hopping behavior with a Verwey-like electronic correlation at around $45 \mathrm{~K}$. The application of the RTA process at $450^{\circ} \mathrm{C}$ results in the destruction of Fe-vacancy order and induces more electron carriers by increasing the $\mathrm{Fe}^{3+}$ valence state. Superconductivity emerges with $T_{\mathrm{C}} \sim 8 \mathrm{~K}$ without changing the chemical stoichiometry of the sample after the RTA process, resulting in the addition of extra carriers in favor of superconductivity.

\section{OPEN ACCESS}

Edited by:

Jun Zhao,

Fudan University, China

Reviewed by:

Jiangang Guo,

Institute of Physics (CAS), China

Meng Wang,

Sun Yat-sen University, China

${ }^{*}$ Correspondence:

Maw-Kuen Wu

mkwu@phys.sinica.edu.tw

Specialty section:

This article was submitted to

Condensed Matter Physics,

a section of the journal

Frontiers in Physics

Received: 29 May 2020 Accepted: 30 September 2020 Published: 13 November 2020

Citation:

Yeh K-Y, Chen Y-R, Lo T-S, WU PM,

Wang M-J, Chang-Liao K-S and WU

M-K (2020) Fe-Vacancy-Ordered

$\mathrm{Fe}_{4} \mathrm{Se}_{5}$ : The Insulating Parent Phase of

FeSe Superconductor.

Front. Phys. 8:567054.

doi: 10.3389/fphy.2020.567054
Keywords: FeSe superconductors, Verwey-like transition, Fe-vacancy order, Mott insulators, Mixed-valence state

\section{INTRODUCTION}

The FeAs-based [1] and FeSe-based [2] superconductors are among the most investigated materials in condensed matter physics since their discovery in 2008. The observation of a wide range of superconducting transition temperatures, with the highest confirmed Cooper pair formation temperature up to $75 \mathrm{~K}$ in monolayer FeSe films [3], provides a unique opportunity to gain more insight into the origin of high-temperature superconductivity. The multiple-orbital nature of the Fe-based materials, combined with spin and charge degrees of freedom, results in the observation of many intriguing phenomena such as structural distortion, magnetic or orbital ordering [4], and electronic nematicity $[5,6]$. There are suggestions that the orbital fluctuation may provide a new channel for realizing superconductivity $[7,8]$.

The parent compounds of FeAs-based materials exhibit structural transitions from a hightemperature tetragonal phase to a low-temperature orthorhombic phase, which is accompanied by an antiferromagnetic (AF) order $[9,10]$. Upon doping, both the orthorhombic structure and the AF phase are suppressed and superconductivity is induced. On the other hand, FeSe undergoes a tetragonal-to-orthorhombic transition at $\sim 90 \mathrm{~K}[2,11,12]$. However, no magnetic order is formed at ambient pressure [12,13] and superconductivity below $\sim 8 \mathrm{~K}[2]$ is crucially related to this orthorhombic distortion. The nematic order coexists with superconductivity but not with longrange magnetic order which has led to arguments that the origin of the nematicity in FeSe is not magnetically but likely orbital-driven [14, 15].

However, recent studies show that the nematic states in the FeSe systems are far more complex [16-20]. There exist strong high-energy spin fluctuations [20] which suggest that the nematicity and magnetism may be still intimately linked. It was also found that there are many interesting features in the band structures of the nematic state. More surprises came as one applied pressure to FeSe. The application of pressure leads to the suppression of structural transition, the appearance of a 
magnetically ordered phase at $\sim 1 \mathrm{GPa}[13,21]$, and $T_{\mathrm{c}}$ increases to a maximum $\sim 37 \mathrm{~K}[22-27]$ at $\sim 6 \mathrm{GPa}$. An even more dramatic enhancement of $T_{c}$ was achieved on monolayer FeSe grown on $\mathrm{SrTiO}_{3}$ substrate [28-31].

The above observations lead to questions that exist since the discovery of FeSe superconductors: what is the exact chemical stoichiometry of the compound and what is the exact phase diagram for the FeSe system? Earlier studies showed that the superconducting property of FeSe is very sensitive to its stoichiometry $[2,12,32]$. The fact that higher superconducting transition temperature exists in monolayer FeSe on $\mathrm{SrTiO}_{3}$ substrate suggests that the commonly accepted phase diagram, derived from assuming that $\mathrm{FeTe}$ is the nonsuperconducting parent compound of FeSe [33], is questionable. Studies have observed the trace of the superconducting feature with $T_{\mathrm{c}}$ close to $40 \mathrm{~K}$ in samples of nanodimensional form [34].

It has been a debate whether there exists an antiferromagnetic Mott insulating parent phase, similar to the cuprate superconductors, for FeSe superconductors [35-37]. Chen et al. first reported the existence of tetragonal $\beta-\mathrm{Fe}_{1-\mathrm{x}} \mathrm{Se}$ with Fe-vacancy orders, characterized by analytical transmission electron microscopy [38]. The authors further argued the $\mathrm{Fe}_{4} \mathrm{Se}_{5}$ phase with $\sqrt{ } 5 \times \sqrt{5} \mathrm{Fe}$-vacancy order to be the parent phase of FeSe superconductors [38]. The Fe-vacancy order observed in the $\mathrm{Fe}_{4} \mathrm{Se}_{5}$ phase is identical to the Fe-vacancy order observed in the $\mathrm{A}_{2} \mathrm{Fe}_{4} \mathrm{Se}_{5}(\mathrm{~A}=\mathrm{K}, \mathrm{Tl}, \mathrm{Rb})$, which has been shown to be an antiferromagnetic [35, 39-42] and is the parent phase of the superconductor $\mathrm{A}_{2-\mathrm{x}} \mathrm{Fe}_{4+\mathrm{x}} \mathrm{Se}_{5}[43,44]$. The detailed studies of the Fe vacancy in $\mathrm{K}_{2} \mathrm{Fe}_{4+\mathrm{x}} \mathrm{Se}_{5}$ reveal that its order/disorder is directly associated with superconductivity. A recent study shows that the Fe-vacancy-ordered $\mathrm{Fe}_{4} \mathrm{Se}_{5}$ nanowire is the nonoxide material with the Verwey-like electronic correlation [45]. It suggests that a charge-ordered state emerges below $T=17 \mathrm{~K}$. The question remains unanswered is whether this Fe-vacancy-ordered phase is the parent compound of superconducting FeSe?

In this paper, we present the results of structure, electrical transport, and magnetic measurements on the polycrystalline sample of $\mathrm{Fe}_{4} \mathrm{Se}_{5}$ treated by rapid-thermal-annealing (RTA) process at a proper temperature and time. After RTA treatment, the sample shows superconductivity with $T_{\mathrm{c}} \sim 8 \mathrm{~K}$ without changing its chemical stoichiometry. Our findings confirm that the $\mathrm{Fe}_{4} \mathrm{Se}_{5}$ with $\mathrm{Fe}$-vacancy order is the parent compound of FeSe superconductors.

\section{EXPERIMENTAL TECHNIQUES}

\section{Sample Preparation}

$\mathrm{Fe}_{4} \mathrm{Se}_{5}$ nanosheets were prepared by a chemical coprecipitation method. First, $200 \mathrm{ml}$ of ethylene glycol was mixed with $\mathrm{NaOH}$ and $\mathrm{SeO}_{2}$ powder and slowly heated up to $160^{\circ} \mathrm{C}$ for mixing well. The volume of $2.4 \mathrm{ml}$ hydrazine hydrate was then added as the reducing agent. Then, at $160^{\circ} \mathrm{C}$, the $\mathrm{Fe}$ precursor solution was added and reacted for $12 \mathrm{~h}$ in order to form $\mathrm{Fe}_{4} \mathrm{Se}_{5}$ nanosheets. The Fe precursor solution is made by dissolving the amount $\mathrm{FeCl}_{2}$ in ethylene glycol. The reaction was done under $\mathrm{N}_{2}$ gas purging to avoid the formation of oxide impurity. To clean the $\mathrm{Fe}_{4} \mathrm{Se}_{5}$ nanosheets, the reacted product was dispersed in acetone with absolute ethanol, and high-speed centrifugation is applied to precipitate the nanosheets and remove the capping ligand dissolved in the above organic solvent. The nanosheets were finally dried in a vacuum for $24 \mathrm{~h}$ and collected. The process for the rapid thermal annealing (RTA) is as follows: the as-grown $\mathrm{Fe}_{4} \mathrm{Se}_{5}$ nanosheets were heated at $450^{\circ} \mathrm{C}$ for $10 \mathrm{~min}$ in a tube furnace with $1 \mathrm{~atm}$ Ar gas inside to maintain a nonoxidation environment as a rapid thermal treatment process. After the rapid thermal treatment, an air-quenching process was taken by flowing room-temperature Ar gas through the tube. All the samples were stored in the oxygen-free glove box.

\section{Analysis}

The crystal structure observation of the $\mathrm{Fe}_{4} \mathrm{Se}_{5}$ samples was carried out by the high-resolution transmission electron microscope (HRTEM, JEOL JEM-2100F) and 4-circle x-ray diffractometer with the incident beam $(12.4 \mathrm{keV})$ of wavelength $0.82656 \AA$ at beam-line BL13A and wavelength $0.61992 \AA$ at beam-line TPS09A in NSRRC. The temperaturedependent structural information of $\mathrm{Fe}_{4} \mathrm{Se}_{5}$ samples was analyzed by the high-resolution neutron powder diffraction (high-resolution powder diffractometer Echidna with the wavelength of $2.4395 \AA$ at ANSTO). The $\mathrm{Fe}_{4} \mathrm{Se}_{5}$ nanosheet powder was pressed into the pallet under $200 \mathrm{~kg} / \mathrm{cm}^{2}$ at $100^{\circ} \mathrm{C}$ for $1 \mathrm{~h}$ for the following measurements: To identify the stoichiometry, the energy-disperse X-ray spectrometer (EDS) setup with the SEM (JEOL JSM-7001F Field Emission Scanning Electron Microscope) was applied. To affirm the valence states of the $\mathrm{Fe}$ ions, the X-ray photoemission spectroscopy (XPS, VG Scientific ESCALAB 250) measurements for the samples were performed. For polycrystalline bulk samples, the resistance was measured by using the standard four-probe method with silver paste for electrical contact and the Hall measurement by a Hall-bar configuration was done by the Quantum Design Physical Properties Measurement System (PPMS, Model 6000). The magnetic property was measured by the Quantum Design Superconducting quantum interference device (SQUID, VSM).

\section{EXPERIMENTAL RESULTS AND DISCUSSIONS}

\section{Structural Analysis of $\mathrm{Fe}_{4} \mathrm{Se}_{5}$}

Figure 1A shows the X-ray diffraction (XRD) patterns of the asgrown $\mathrm{Fe}_{4} \mathrm{Se}_{5}$ sample at room temperature. The diffraction pattern, which exhibits superstructure peaks, is refined with a tetragonal $P 4$ symmetry with $\sqrt{ } 5 \times \sqrt{ } 5$ Fe-vacancy order instead of the tetragonal $P 4 / \mathrm{mmm}$ symmetry as observed in FeSe [2]. The insets are the TEM image of as-grown $\mathrm{Fe}_{4} \mathrm{Se}_{5}$ nanocrystal and its TEM-SAED (selective area electron diffraction) patterns along the $c$-axis. The observation of extra diffraction points among the main diffraction points in the SAED pattern confirms the $\sqrt{ } 5 \times$ $\sqrt{ } 5 \mathrm{Fe}$-vacancy order in the as-grown $\mathrm{Fe}_{4} \mathrm{Se}_{5}$ nanocrystal [35]. After RTA treatment at $450^{\circ} \mathrm{C}$, the superstructure peaks observed 

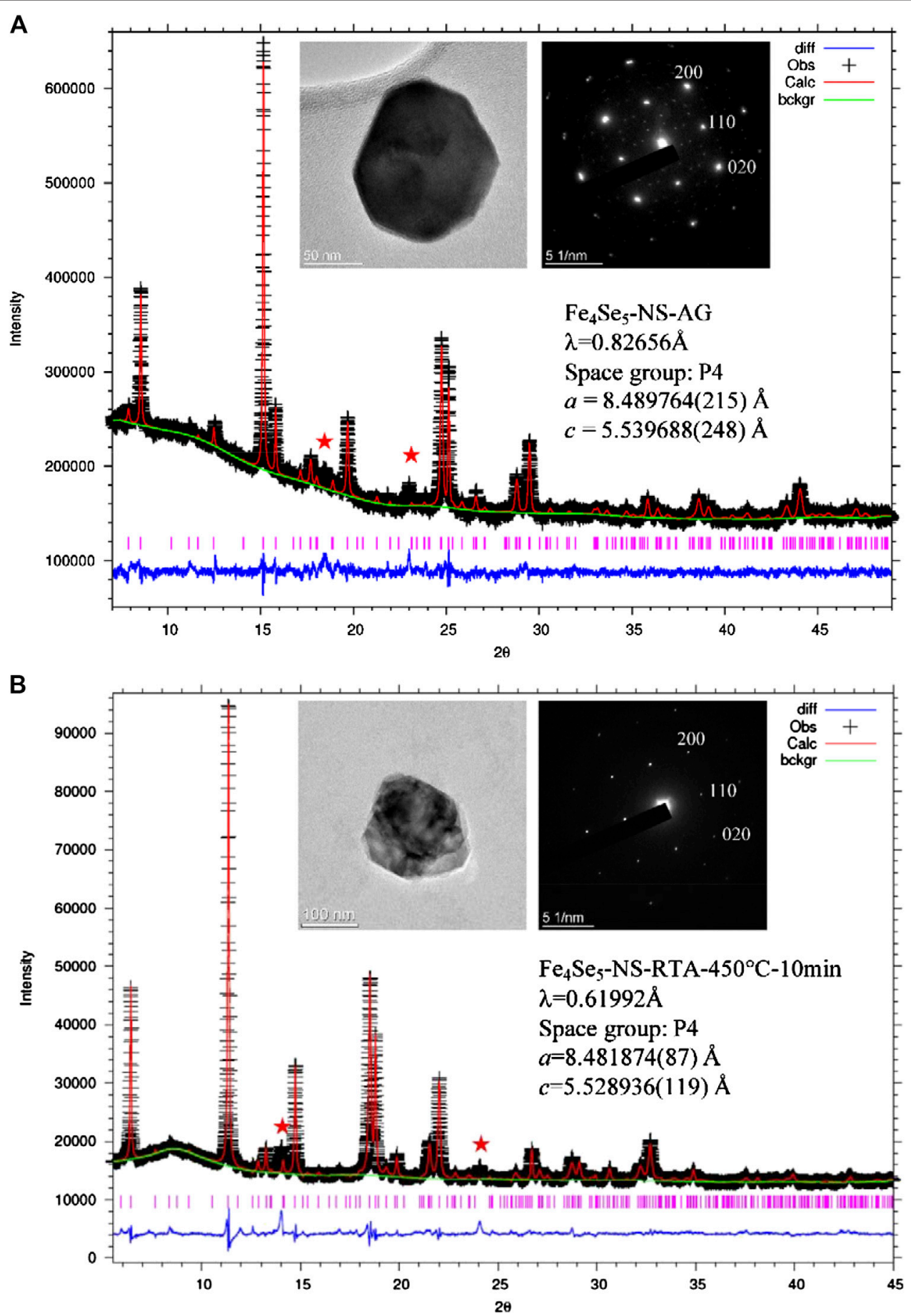

FIGURE 1 |X-ray diffraction patterns and their Rietveld refinement results of $\mathbf{( A )}$ the as-grown $\mathrm{Fe}_{4} \mathrm{Se}_{5}$ nanocrystals and (B) the sample after the RTA process at $450^{\circ} \mathrm{C}$. The insets in (A) and (B) show the transmission electron microscope (TEM) images and selective area electron diffraction (SAED) patterns of the samples, respectively.

in XRD and TEM-SAED due to the $\sqrt{5} \times \sqrt{ } 5$ Fe-vacancy order disappear, as shown in Figure 1B and its inset. The refinement results, using the same $P 4$ symmetry, reveal that the occupations of Fe at vacancy $4 \mathrm{~d}$ sites and the originally occupied 16i sites are almost the same, indicating that the $\mathrm{Fe}$ vacancies become disordered after RTA treatment. It is noted that the XRD patterns of the RTA treatment sample are nearly ten times smaller in the intensity response. This is due to the different synchrotron beamlines we used for our measurements. However, the difference does not affect the refined results. 

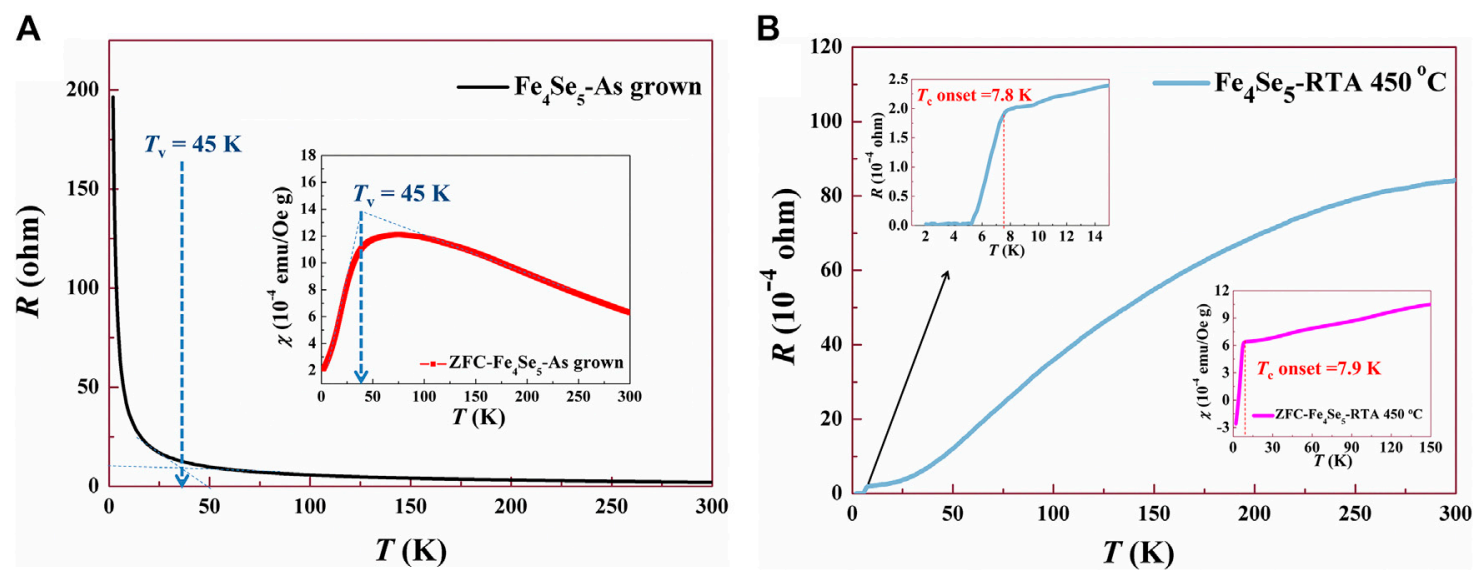

FIGURE 2 | (A) Temperature-dependent resistance of the as-grown $\mathrm{Fe}_{4} \mathrm{Se}_{5}$ sample showing a metal-insulator transition $T_{V}$ with the onset of resistance rise at $\sim 45 \mathrm{~K}$. The inset is the magnetic susceptibility from 300 to $2 \mathrm{~K}$ of the same sample, which shows a large drop in susceptibility with the onset temperature also at $\sim 45 \mathrm{~K}$. (B) Temperature-dependent resistance of the $\mathrm{Fe}_{4} \mathrm{Se}_{5}$ sample after $450^{\circ} \mathrm{C}$ RTA process. The sample becomes metallic and shows superconducting transition with the onset $T_{\mathrm{c}} \sim 7.8 \mathrm{~K}$, as highlighted in the upper-left inset. The lower-right inset is the magnetic susceptibility which further demonstrates the superconducting transition with $T_{\mathrm{c}} \sim 7.9 \mathrm{~K}$.

It is noted that the $\mathrm{FeSe}_{4}$ tetrahedron in as-grown $\mathrm{Fe}_{4} \mathrm{Se}_{5}$ is highly distorted due to the existence of the Fe-vacancy order. As the Fe vacancies disordered by the RTA treatment, the FeSe4 tetrahedron becomes more symmetric. The refined structure parameters are tabulated in Supplementary Tables S1 and S2. The EDS analysis confirms that the stoichiometries of samples keeps $\mathrm{Fe}_{4} \mathrm{Se}_{5}$ with Fe/Se ratio of $44.5: 55.5$ and $45.3: 54.7$ before and after the RTA treatment, respectively, as shown in Supplementary Figure S2 in the supplementary information.

\section{Temperature Dependence of the Resistance and the Magnetic Susceptibility Measurement of $\mathrm{Fe}_{4} \mathrm{Se}_{5}$}

Figure 2A shows the temperature dependence of the resistance for the as-grown polycrystalline pellet sample made of $\mathrm{Fe}_{4} \mathrm{Se}_{5}$ nanosheets. The inset is the magnetic susceptibility of the asgrown sample from 300 to $2 \mathrm{~K}$. The R-T results of $\mathrm{Fe}_{4} \mathrm{Se}_{5}$ exhibit a metal-insulator transition with the sharp rise in resistance at $\sim 45 \mathrm{~K}$. The data can be well-fitted to the three-dimensional Mott variable-range-hopping model (3D-MVH): $\rho(T)=\rho_{0} \exp \left(T_{0} / T\right)^{v}$, where $T_{0}$ is the variable-range-hopping characteristic temperature, and the exponent $v$ is $1 /(d+1)$ with $d=3$ (the fitting results are shown in Supplementary Figure S2. A transition temperature $T_{\mathrm{V}}$ is marked as the onset temperature of 3D-MVH behavior). The variable-range-hopping characteristic temperature $T_{0}$ calculated is $\sim 1,400 \mathrm{~K}$ for the asgrown sample. The magnetic susceptibility of the same sample shows paramagnetic behavior as the sample cools down from $300 \mathrm{~K}$, and a sudden drop in susceptibility appears at about the same temperature as the resistance transition temperature $\left(T_{\mathrm{V}}\right)$ $\sim 45 \mathrm{~K}$. The sharp resistive rise and the diamagnetic drop are the two signatures for the Verwey transition observed in $\mathrm{Fe}_{3} \mathrm{O}_{4}$, which occurs at $125 \mathrm{~K}$. These results are also in line with those reported results in the $\mathrm{Fe}_{4} \mathrm{Se}_{5}$ nanowire, which was recently demonstrated to exhibit the Verwey-like electronic correlation [45].

Figure 2B shows the temperature-dependence resistance for the $\mathrm{Fe}_{4} \mathrm{Se}_{5}$ samples after $300^{\circ}$ and $450 \mathrm{C}$ RTA process. The sample after $300^{\circ} \mathrm{C}$ remains to behave like semiconductor. The sample treated at $450^{\circ}$ and $300^{\circ} \mathrm{C}$ changes to metallic and becomes superconducting below $\sim 5 \mathrm{~K}$ with the onset superconductive critical temperature $\left(T_{\mathrm{c}}\right) \sim 7.8 \mathrm{~K}$, as evidenced in the upper-left inset of Figure $\mathbf{2 B}$. The lower-right inset is the magnetic susceptibility, which further demonstrates the superconducting transition with onset $T_{\mathrm{c}} \sim 7.9 \mathrm{~K}$. The evolution to superconductivity in this RTA-treated sample is similar to that reported in the $\mathrm{K}_{2-\mathrm{x}} \mathrm{Fe}_{4+\mathrm{y}} \mathrm{Se}_{5}$ system, where superconductivity appears after Fe vacancies becoming disordered through hightemperature annealing and rapid quenching processes $[43,44]$.

\section{XPS and Hall Measurement of $\mathrm{Fe}_{4} \mathrm{Se}_{5}$}

In order to gain more insight into the observed Verwey-like electronic correlation, XPS at room temperature and temperature-dependent Hall measurements on the samples were performed. Figure $\mathbf{3 A}$ is the observed XPS results for the as-grown $\mathrm{Fe}_{4} \mathrm{Se}_{5}$ sample. Figure 3A is the observed XPS results for the as-grown $\mathrm{Fe}_{4} \mathrm{Se}_{5}$ sample. The XPS spectrum clearly reveals two peaks showing the existence of mixed-valence states of Fe. The observed two peaks, at 708.5 and $711.5 \mathrm{eV}$, can be associated with the $\mathrm{Fe}^{2+}$ and $\mathrm{Fe}^{3+}$ states, respectively. The best data fitting gives the ratio between $\mathrm{Fe}^{2+}$ and $\mathrm{Fe}^{3+}$ close to $1: 1$. This result is similar to that observed in the magnetite $\mathrm{Fe}_{3} \mathrm{O}_{4}$.

Figure 3B displays the XPS results for samples with RTA treated at $300^{\circ}$ and $450^{\circ} \mathrm{C}$. After the RTA treatment, the $\mathrm{Fe}^{3+}$ state becomes dominant. The extracted $\mathrm{Fe}^{3+}$ ion to total $\mathrm{Fe}$ atoms ratio is $58.7 \%$ for $300^{\circ} \mathrm{C}$ RTA-treated and $73.2 \%$ for $450^{\circ} \mathrm{C}$ RTA-treated samples, respectively, indicating a substantial increase in electron carriers in these samples. It should be noted that the sample after $300^{\circ} \mathrm{C}$ still exhibits temperature-dependent behavior like 

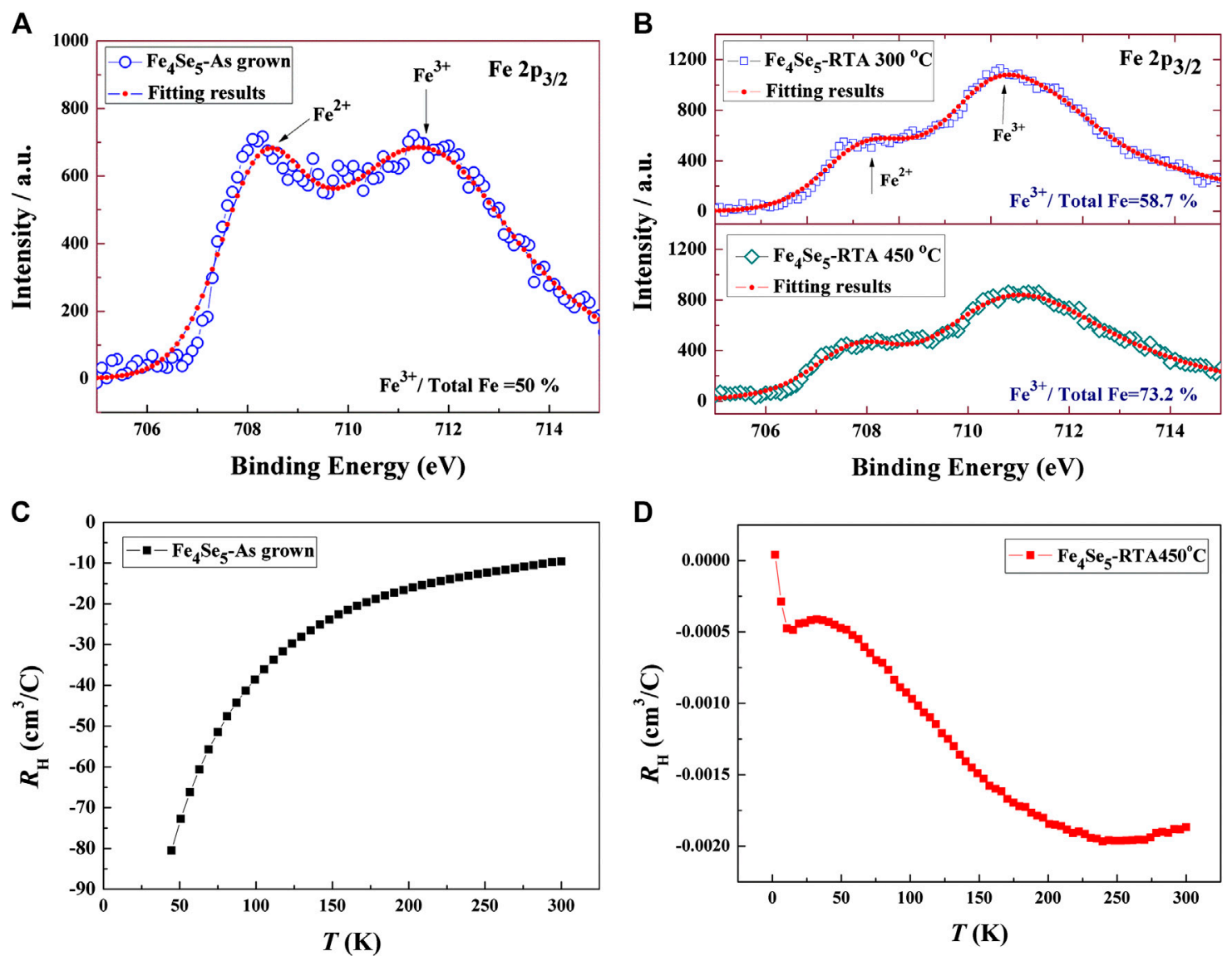

FIGURE 3 | $\mathrm{Fe}_{2 \mathrm{p} 3 / 2}$ spectrum of XPS analysis for (A) the as-grown $\mathrm{Fe}_{4} \mathrm{Se}_{5}$ sample and (B) the $300^{\circ}$ and $450^{\circ} \mathrm{C} \mathrm{RTA-treated} \mathrm{Fe} \mathrm{Se}_{5}$ sample. The temperaturedependent Hall coefficient for (C) the as-grown $\mathrm{Fe}_{4} \mathrm{Se}_{5}$ sample and (D) the $450^{\circ} \mathrm{C}$ RTA-treated $\mathrm{Fe}_{4} \mathrm{Se}_{5}$ sample.

semiconductor. There is no specific difference of Se $3 \mathrm{~d}$ peak at $54.7 \mathrm{eV}$ before and after the RTA process of the $\mathrm{Fe}_{4} \mathrm{Se}_{5}$ sample according to the XPS result, as shown in Supplementary Figure S3.

It is known that tetragonal FeSe is a metal with two-band based on the first-principles electronic structure calculation, for example, by T. Xiang et al., [46]. T. Xiang et al. also reported that the electronic structure of $\mathrm{Fe}_{4} \mathrm{Se}_{5}$ with $\sqrt{5} \times \sqrt{ } 5 \mathrm{Fe}$-vacancy order is a pair checkboard antiferromagnetic insulator. The calculation shows that the Fe-vacancy-ordered $\mathrm{Fe}_{4} \mathrm{Se}_{5}$ has a single-band structure with n-type carrier dominated and a bandgap $290 \mathrm{meV}$. Berlijn et al. [47] investigated the effect of disordered Fe vacancies on the normal-state electronic structure of the alkali-intercalated FeSe system, where the $\mathrm{KFe}_{4} \mathrm{Se}_{5}$ exhibits exactly the same $\mathrm{Fe}$-vacancy order as that in $\mathrm{Fe}_{4} \mathrm{Se}_{5}$. They found that the disorder of Fe-vacancy can effectively raise the chemical potential giving enlarged electron pockets without adding carriers to the system.

It is noted that, as reported by Chen et al. [38], there exists a series of $\mathrm{Fe}_{\mathrm{x}} \mathrm{Se}_{\mathrm{y}}$ compounds with $\mathrm{x} / \mathrm{y}=1 / 2,2 / 3,3 / 4,4 / 5$, etc. We have carried out a systematic study using the coprecipitation method to successfully prepare tetragonal $\mathrm{Fe}_{(1-\mathrm{x})} \mathrm{Se}$ with stoichiometry of $\mathrm{Fe}_{3} \mathrm{Se}_{4}$ and $\mathrm{Fe}_{4} \mathrm{Se}_{5}$. Based on the XPS results, the observed $\mathrm{Fe}^{3+} / \mathrm{Fe}^{2+}$ ratio is
2 and 1 for tetragonal $\mathrm{Fe}_{3} \mathrm{Se}_{4}$ and $\mathrm{Fe}_{4} \mathrm{Se}_{5}$, respectively, as shown in Supplementary Figure S4A and Figure 3A. These data imply that $\mathrm{Fe}_{3} \mathrm{Se}_{4}$ would be hole-doped and $\mathrm{Fe}_{4} \mathrm{Se}_{5}$ be electron-doped if there are additional carriers based on the simple charge balance picture by considering $\mathrm{Fe}_{3} \mathrm{Se}_{4}$ to be the combination of $\mathrm{Fe}^{(2+)} \mathrm{Se}$ and $\mathrm{Fe}_{2}{ }^{(3+)} \mathrm{Se}_{3}$, whereas $\mathrm{Fe}_{4} \mathrm{Se}_{5}$ is from $2\left(\mathrm{Fe}^{(2+)} \mathrm{Se}\right)$ and $\mathrm{Fe}_{2}{ }^{(3+)} \mathrm{Se}_{3}$. Indeed, our Hall measurement results show at $300 \mathrm{~K}$ a hole concentration of $1.20 \times$ $10^{19} / \mathrm{cm}^{3}$ for $\mathrm{Fe}_{3} \mathrm{Se}_{4}$ (Supplementary Figure S4B) and electron concentration of $-6.52 \times 10^{17} / \mathrm{cm}^{3}$ for $\mathrm{Fe}_{4} \mathrm{Se}_{5}$.

Both of the as-grown and RTA-treated $\mathrm{Fe}_{4} \mathrm{Se}_{5}$ show a single-band behavior with n-type carrier from the Hall resistivity measurements, as shown in Supplementary Figure S5. The Hall coefficient of the as-grown sample at room temperature is $-9.59 \mathrm{~cm}^{3} / \mathrm{C}$, corresponding to the electron carrier concentration of $6.52 \times$ $10^{17} \mathrm{~cm}^{-3}$, and the carrier concentration decreases by about a factor of 8 at the transition temperature $T_{\mathrm{V}}$, as shown in Figure 3C.

After the $\mathrm{Fe}_{4} \mathrm{Se}_{5}$ sample is RTA-treated at $450{ }^{\circ} \mathrm{C}$, the $\mathrm{Fe}^{3+} / \mathrm{Fe}^{2+}$ ratio becomes close to $3: 1$, which means that a large number of electrons are introduced and subsequently induced superconductivity. Indeed, the Hall measurement results, as shown in Figure 3D, show that the carrier concentration at $300 \mathrm{~K}$ increases to $-3.34 \times 10^{21} / \mathrm{cm}^{3}$ (Hall coefficient $-1.87 \times$ $10^{-3} \mathrm{~cm}^{3} / \mathrm{C}$ ) for $450 \mathrm{C}$ RTA-treated sample. The electron carrier 


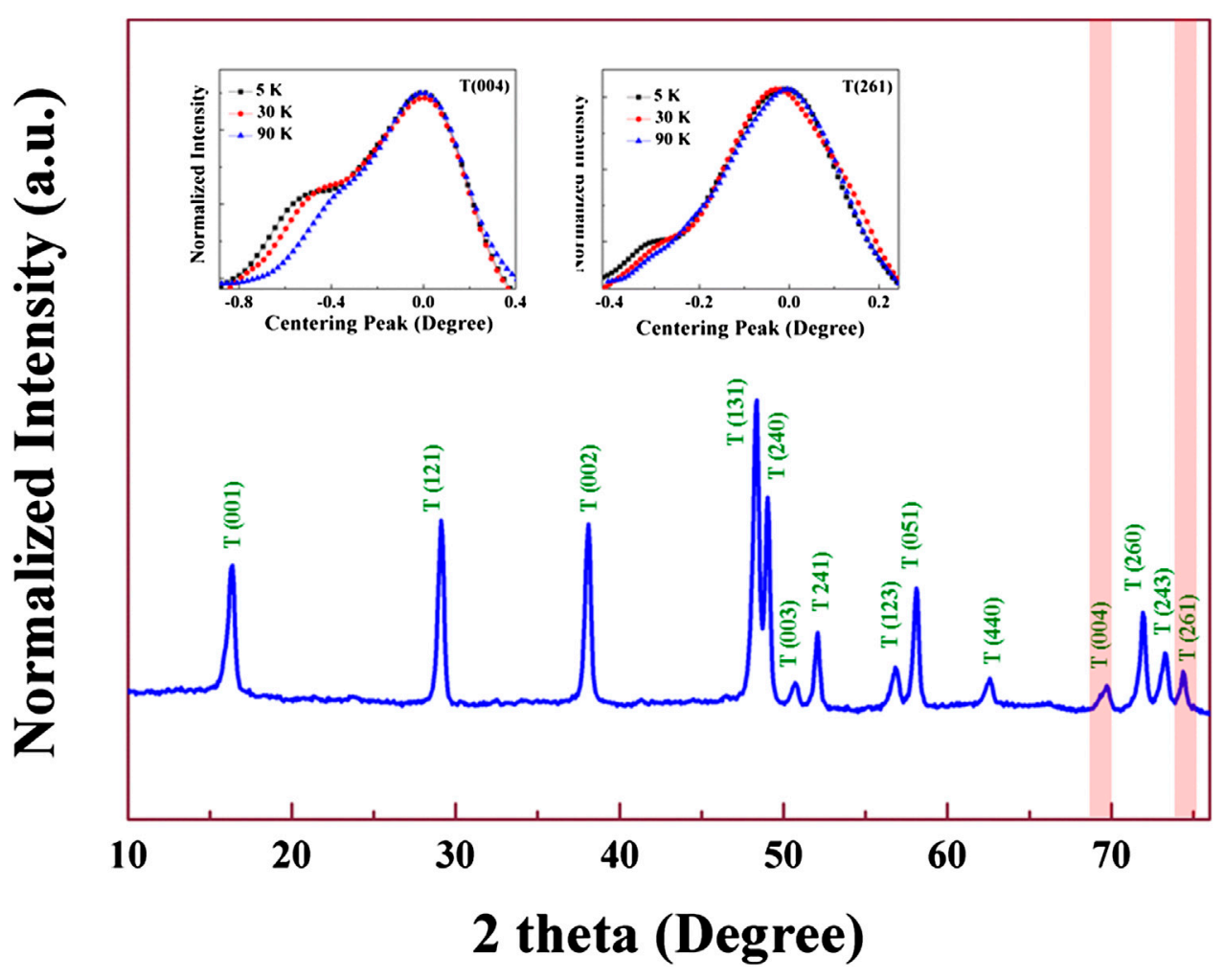

FIGURE 4 | The neutron diffraction patterns of as-grown $\mathrm{Fe}_{4} \mathrm{Se}_{5}$ nanosheet at $300 \mathrm{~K}$. The tetragonal $P 4$ symmetry is identified. The insets are the diffraction peaks of (004) and (261) at low temperatures showing the growth of additional peaks, indicating a structural change at low temperature.

concentration is about four orders of magnitude increase comparing with the as-grown $\mathrm{Fe}_{4} \mathrm{Se}_{5}$. Obviously, the RTA treatment disrupts the Fe-vacancy long-range order and leads to the increase of electron carriers.

\section{Neutron Diffraction of $\mathrm{Fe}_{4} \mathrm{Se}_{5}$}

It is well known that the Verwey transition in magnetite exhibits a structural transition accompanied by the sharp resistive and magnetic susceptibility changes. To examine whether such a structural change exists for the as-grown $\mathrm{Fe}_{4} \mathrm{Se}_{5}$, we have carried out the neutron diffraction at low temperatures.

The detailed structural information of the as-grown $\mathrm{Fe}_{4} \mathrm{Se}_{5}$ sample measured by neutron diffraction at different temperatures is shown in Figure 4. At room temperature, the neutron data, consistent with XRD results, fit well with the P4-tetragonal symmetry. At low temperatures, a distortion appears at temperatures below $30 \mathrm{~K}$. The data at $5 \mathrm{~K}$, with evident peak emergence shown in the inset of Figure 4, indicates a possible structural change. This result further supports that the as-grown $\mathrm{Fe}_{4} \mathrm{Se}_{5}$ nanosheets, similar to the results observed in $\mathrm{Fe}_{4} \mathrm{Se}_{5}$ nanowire, show the Verwey-like correlation. The Verwey-like transition temperature of $\sim 45 \mathrm{~K}$ in nanosheets is higher than that observed in the nanowire, which was found to be $\sim 30 \mathrm{~K}$. This shows the size dependence of $T_{\mathrm{V}}$, which is also noticed in Verwey transition [48-50]. Currently, we are waiting for the results of the detailed high-resolution XRD at low temperatures using a synchrotron source to determine exactly the low-temperature phase and the transition temperature.

\section{CONCLUSION}

We carried out a detailed study to investigate whether there exists an insulating parent phase for FeSe superconductors. Our studies unambiguously show that 1 ) the $\sqrt{ } 5 \times \sqrt{ } 5$ Fe-vacancyordered $\mathrm{Fe}_{4} \mathrm{Se}_{5}$ is a Mott insulator with Verwey-like transition at low temperature; 2) $\mathrm{Fe}_{4} \mathrm{Se}_{5}$ is the parent compound of the FeSe superconductors. The application of the RTA process at $450^{\circ} \mathrm{C}$ disrupts Fe-vacancy order and induces more electron carriers by increasing the $\mathrm{Fe}^{3+}$ valence state. Superconductivity emerges with $T_{\mathrm{c}} \sim 8 \mathrm{~K}$ without changing the chemical stoichiometry of the sample after the RTA process. Consistent with the observations in $\mathrm{K}_{2} \mathrm{Fe}_{4+\mathrm{x}} \mathrm{Se}_{5}$, superconductivity is directly related to the disappearance of Fe-vacancy long-range order. In the $\mathrm{Fe}_{4} \mathrm{Se}_{5}$ case, no extra Fe doping is required as the random occupation of $\mathrm{Fe}$ atom in the vacancy sites, resulting in the addition of extra carriers in favor of superconductivity. More detailed evolution of superconductivity by varying the RTA temperature and time is currently underway in order to gain more insight into the exact phase diagram of the FeSe superconductors.

\section{DATA AVAILABILITY STATEMENT}

The raw data supporting the conclusions of this article will be made available by the authors, without undue reservation. 


\section{AUTHOR CONTRIBUTIONS}

MJW and MKW designed research. KYY, TSL, and YRC performed research. MJW and KSCL contributed new reagents/analytic tools. KYY, TSL, PMW, YRC, KSCL, MJW, and MKW analyzed data and took part in physics discussions. KYY, MJW, TSL, PMW, and MKW wrote the paper.

\section{FUNDING}

The work is supported by the Ministry of Science and Technology under Grant No. MOST108-2633-M-001-001 and Academia Sinica Thematic Research Grant No. AS-TP-106-M01.

\section{REFERENCES}

1. Kamihara $\mathrm{Y}$, Watanabe $\mathrm{T}$, Hirano $\mathrm{M}$, Hosono $\mathrm{H}$. Iron-based layered superconductor $\mathrm{La}\left[\mathrm{O}_{1-\mathrm{x}} \mathrm{F}_{\mathrm{x}}\right] \mathrm{FeAs}(\mathrm{x}=0.05-0.12)$ with $T_{\mathrm{c}}=26 \mathrm{~K}$. J Am Chem Soc (2008) 130:3296. doi:10.1021/ja800073m

2. Hsu FC, Luo JY, Yeh KW, Chen TK, Huang TW, Wu PM, et al.. Superconductivity in the PbO-type structure a-FeSe. Proc Natl Acad Sci USA (2008) 105:14262. doi:10.1073/pnas.0807325105

3. Peng R, Xu HC, Tan SY, Cao H, Xia M, Shen XP, et al.. Tuning the band structure and superconductivity in single-layer FeSe by interface engineering. Nat Commun (2014) 5:5044. doi:10.1038/ncomms6044

4. Yi M, Zhang Y, Shen ZX, Lu D. Role of the orbital degree of freedom in ironbased Superconductors. Npj Quantum Mater (2017) 2:57. doi:10.1038/s41535017-0059-y

5. Fernandes RM, Chubukov AV, Schmalian J. Magnetically driven suppression of nematic order in an iron-based superconductor. Nat Phys (2014) 10:97. doi:10. 1038/NPHYS2877

6. Yu R, Zhu JX, Si Q. Orbital selectivity enhanced by nematic order in FeSe. Phys Rev Lett (2018) 121:227003. doi:10.1103/PhysRevLett.121.227003

7. Saito T, Onari S, Kontani H. Orbital fluctuation theory in iron pnictides: effects of As-Fe-As bond angle, isotope substitution, and $Z_{2}$-orbital pocket on superconductivity. Phys. Rev. B (2010) 82:144510. doi:10.1103/PhysRevB.82.144510

8. Kontani H, Onari S. Orbital-fluctuation-mediated superconductivity in iron pnictides: analysis of the five-orbital Hubbard-Holstein model. Phys Rev Lett (2010) 104:157001. doi:10.1103/PhysRevLett.104.157001

9. Yu W, Aczel AA, Williams TJ, Bud'ko SL, Ni N, Canfield PC, et al.. Absence of superconductivity in single-phase $\mathrm{CaFe}_{2} \mathrm{As}_{2}$ under hydrostatic pressure. Phys Rev B (2009) 79:020511. doi:10.1103/PhysRevB.79.020511

10. Goldman AI, Argyriou DN, Ouladdiaf B, Chatterii T, Kreyssig A, Nandi S, et al.. Lattice and magnetic instabilities in $\mathrm{CaFe}_{2} \mathrm{As}_{2}$ : a single-crystal neutron diffraction study. Phys Rev B (2008) 78:100506. doi:10.1103/PhysRevB.78.100506

11. Margadonna S, Takabayashi Y, McDonald MT, Kasperkiewicz K, Mizuguchi Y, Takano Y, et al.. Crystal structure of the new $\mathrm{FeSe}_{1-\mathrm{x}}$ superconductor. Chem. Commun (2008) 0:5607. doi:10.1039/b813076k

12. McQueen TM, Williams AJ, Stephens PW, Tao J, Zhu Y, Ksenofontov V, et al.. Tetragonal-to-orthorhombic structural phase transition at $90 \mathrm{~K}$ in the superconductor $\mathrm{Fe}_{1.01} \mathrm{Se}$. Phys Rev Lett (2009) 103:057002. doi:10.1103/ PhysRevLett.103.057002

13. Bendele M, Amato A, Conder K, Elender M, Keller H, Klauss H-H, et al.. Pressure induced static magnetic order in superconducting $\mathrm{FeSe}_{1-\mathrm{x}}$. Phys Rev Lett (2010) 104:087003. doi:10.1103/PhysRevLett.104.087003

14. Baek SH, Efremov DV, Ok JM, Kim JS, Brink JVD, Bchner B. Orbitaldriven nematicity in FeSe. Nat Mater (2015) 14:210. doi:10.1038/ NMAT4138

15. Bohmer AE, Arai T, Hardy F, Hattori T, Iye T, Wolf T, et al.. Origin of the tetragonal-to-orthorhombic phase transition in FeSe:A combined thermodynamic and NMR study of nematicity. Phys Rev Lett (2015) 114: 027001. doi:10.1103/PhysRevLett.114.027001

\section{ACKNOWLEDGMENTS}

The authors appreciate very much the help from Dr. G. T Huang for synchrotron XRD measurements and Dr. C. P. Yen for the analysis of XPS results. We thank the technical support from NanoCore, the Core Facilities for Nanoscience and Nanotechnology at Academia Sinica in Taiwan.

\section{SUPPLEMENTARY MATERIAL}

The Supplementary Material for this article can be found online at: https://www.frontiersin.org/articles/10.3389/fphy. 2020.567054/full\#supplementary-material

16. Chubukov AV, Fernandes RM, Schmalian J. Origin of nematic order in FeSe. Phys Rev B (2015) 91:201105. doi:10.1103/PhysRevB.91.201105

17. Glasbrenner JK, MazinII, Jeschke HO, Hirschfeld PJ, Fernandes RM, Valentł R. Effect of magnetic frustration on nematicity and superconductivity in iron chalcogenides. Nat Phys (2015) 11:953. doi:10.1038/NPHYS3434

18. Wang F, Kivelson SA, Lee DH. Nematicity and quantum paramagnetism in FeSe. Nat Phys (2015) 11:959. doi:10.1038/NPHYS3456

19. Yu R, Si QM. Antiferroquadrupolar and ising-nematic orders of a frustrated bilinear-biquadratic heisenberg model and implications for the magnetism of FeSe. Phys Rev Lett (2015) 115:116401. doi:10.1103/ PhysRevLett.115.116401

20. Wang QS, Shen Y, Pan BY, Hao YO, Ma MW, Zhou F, et al.. Strong interplay between stripe spin fluctuations, nematicity and superconductivity in FeSe. Nat Mater (2016) 15:159. doi:10.1038/NMAT4492

21. Bendele M, Ichsanow A, Pashkevich Y, Keller L, Strässle Th, Gusev A, et al.. Coexistence of superconductivity and magnetism in $\mathrm{FeSe}_{1-\mathrm{x}}$ under pressure. Phys. Rev. B (2012) 85:064517. doi:10.1103/PhysRevB.85.064517

22. Mizuguchi Y, Tomioka F, Tsuda S, Yamaguchi T, Takano Y. Superconductivity at $27 \mathrm{~K}$ in tetragonal FeSe under high pressure. Appl. Phys. Lett (2008) 93: 152505. doi:10.1063/1.3000616

23. Medvedev S, McQueen T, Troyan I, Palasyuk T, Eremets M, Cava R, et al. Electronic and magnetic phase diagram of $\beta-\mathrm{Fe}_{1.01}$ with superconductivity at 36.7 K under pressure. Nat. Mater (2009) 8:630. doi:10.1038/NMAT2491

24. Margadonna S, Takabayashi Y, Ohishi Y, Mizuguchi Y, Takano Y, Kagayama T, et al. Pressure evolution of the low-temperature crystal structure and bonding of the superconductor FeSe $\left(T_{\mathrm{c}}=37 \mathrm{~K}\right)$. Phys. Rev. B (2009) 80: 064506. doi:10.1103/PhysRevB.80.064506

25. Garbarino G, Sow A, Lejay P, Sulpice A, Toulemonde P, Mezouar M, et al. High-temperature superconductivity $\left(T_{\mathrm{c}}\right.$ onset at $\left.34 \mathrm{~K}\right)$ in the high-pressure orthorhombic phase of FeSe. Europhys Lett (2009) :86. doi:10.1209/0295-5075/ $86 / 27001$

26. Masaki S, Kotegawa H, Hara Y, Tou H, Murata K, Mizuguchi Y, et al. Precise pressure dependence of the superconducting transition temperature of FeSe: resistivity and 77Se-NMR study. J Phys Soc Jpn (2009) 78:063704. doi:10.1143/ JPSJ.78.063704

27. Okabe H, Takeshita N, Horigane K, Muranaka T, Akimitsu J. Pressureinduced high- $T_{\mathrm{c}}$ superconducting phase in FeSe: correlation between anion height and $T_{\mathrm{c}}$. Phys Rev B (2010) 81:205119. doi:10.1103/ PhysRevB.81.205119

28. Wang QY, Li Z, Zhang WH, Zhang ZC, Zhang JS, Li W, et al. Interfaceinduced high-temperature superconductivity in single unit-cell FeSe films on $\mathrm{SrTiO}_{3}$. Chin Phys Lett (2012) 29:037402. doi:10.1088/0256-307X/29/3/ 037402

29. Liu DF, Zhang WH, Mou DX, He JF, Ou YB, Wang QY, et al. Electronic origin of high-temperature superconductivity in single-layer FeSe superconductor. Nat Commun (2012) 3:931. doi:10.1038/ncomms1946

30. He SL, He JF, Zhang WH, Zhao L, Liu DF, Liu X, et al. Phase diagram and electronic indication of high-temperature superconductivity at $65 \mathrm{~K}$ in singlelayer FeSe films. Nat Mater (2013) 12:605. doi:10.1038/NMAT3648 
31. Tan SY, Zhang Y, Xia M, Ye ZR, Chen F, Xie X, et al. Interface-induced superconductivity and strain-dependent spin density waves in $\mathrm{FeSe} / \mathrm{SrTiO}_{3}$ thin films. Nat Mater (2013) 12:634. doi:10.1038/NMAT3654

32. McQueen TM, Huang Q, Ksenofontov V, Felser C, Xu Q, Zandbergen H, et al. Extreme sensitivity of superconductivity to stoichiometry in $\mathrm{Fe}_{1+\delta} \mathrm{Se}$. Phys Rev $B$ (2009) 79(1):014522. doi:10.1103/PhysRevB.79.014522

33. Liu TJ, Hu J, Qian B, Fobes D, Mao ZQ, Bao W, et al. From $(\pi, 0)$ magnetic order to superconductivity with $(\pi, \pi)$ magnetic resonance in $\mathrm{Fe}_{1}, 02 \mathrm{Te} 1-\mathrm{xSex}$. Nat Mater (2010) 9:718. doi:10.1038/NMAT2800

34. Chang $\mathrm{CC}$, Wang $\mathrm{CH}$, Wen $\mathrm{MH}$, Wu YR, Hsieh YT, Wu MK. Superconductivity in $\mathrm{PbO}$-type tetragonal FeSe nanoparticles. Solid State Commun (2012) 152:649. doi:10.1016/j.ssc.2012.01.030

35. Zhou Y, Xu DH, Zhang FC, Chen WQ. Theory for superconductivity in (Tl,K)FexSe2 as a doped Mott insulator. Europhys Lett (2011) 95:17003. doi:10.1209/0295-5075/95/17003

36. Yu R, Zhu JX, Si Q. Mott transition in modulated lattices and parent insulator of (K; Tl) ${ }_{\mathrm{y}} \mathrm{Fe}_{\mathrm{x}} \mathrm{Se}_{2}$ superconductors. Phys Rev Lett (2011) 106:186401. doi:10. 1103/PhysRevLett.106.186401

37. Zhao X, Ma F, Lu ZY, Xiang T. $A \mathrm{FeSe}_{2}(A=\mathrm{Tl}, \mathrm{K}, \mathrm{Rb}$, or Cs): iron-based superconducting analog of the cuprates. Phys Rev B (2020) 101:184504. doi:10. 1103/PhysRevB.101.184504

38. Chen TK, Chang CC, Chang HH, Fang AH, Wang $\mathrm{CH}$, Chao WH, et al. Fevacancy order and superconductivity in tetragonal $\beta-\mathrm{Fe}_{1-\mathrm{x}} \mathrm{Se}$. Proc Natl Acad Sci USA (2014) 111:63. doi:10.1073/pnas.1321160111

39. Bao W, Huang QZ, Chen GF, Green MA, Wang DM, He JB, et al. A novel large moment antiferromagnetic order in $\mathrm{K}_{0.8} \mathrm{Fe}_{1.6} \mathrm{Se}_{2}$ superconductor. Chin Phys Lett (2011) 28:086104. doi:10.1088/0256-307X/28/8/086104

40. Zhao J, Cao H, Bourret-Courchesne E, Lee DH, Birgeneau RJ. Neutrondiffraction measurements of an antiferromagnetic semiconducting phase in the vicinity of the high-temperature superconducting state of $\mathrm{K}_{\mathrm{x}} \mathrm{Fe}_{2-\mathrm{y}} \mathrm{Se}_{2}$. Phys Rev Lett (2012) 109:267003. doi:10.1103/PhysRevLett. 109.267003

41. Liu RH, Luo XG, Zhang M, Wang AF, Ying JJ, Wang XF, et al. Coexistence of superconductivity and antiferromagnetism in single crystals $\mathrm{A}_{0.8} \mathrm{Fe}_{2-\mathrm{y}} \mathrm{Se}_{2}$ ( $\mathrm{A}=\mathrm{K}, \mathrm{Rb}, \mathrm{Cs}, \mathrm{Tl} / \mathrm{K}$ and $\mathrm{Tl} / \mathrm{Rb}$ ): evidence from magnetization and resistivity. Europhys Lett (2011) 94:27708. doi:10.1209/0295-5075/94/27008

42. Fang MH, Wang HD, Dong CH, Li ZJ, Feng CM, Chen J, et al. Fe-based superconductivity with $\mathrm{T}_{\mathrm{c}}=31 \mathrm{~K}$ bordering an antiferromagnetic insulator in (Tl,K) $\mathrm{Fe}_{\mathrm{x}} \mathrm{Se}_{2}$. Europhys Lett (2011) 94:27009. doi:10.1209/0295$5075 / 94 / 27009$
43. Wang CH, Chen TK, Chang CC, Hsu CH, Lee YC, Wang MJ, et al. Disordered $\mathrm{Fe}$ vacancies and superconductivity in potassium-intercalated iron selenide $\left(\mathrm{K}_{2-\mathrm{x}} \mathrm{Fe}_{4+\mathrm{y}} \mathrm{Se}_{5}\right)$. Europhys Lett (2015) 111:27004. doi:10.1209/0295-5075/111/ 27004

44. Wang CH, Lee CC, Huang GT, Yang JY, Wang MJ, Sheu HS, et al. Role of the extra Fe in $\mathrm{K}_{2-\mathrm{x}} \mathrm{Fe}_{4+\mathrm{y}} \mathrm{Se}_{5}$ superconductors. Proc Natl Acad Sci USA (2019) 116: 1104. doi:10.1073/pnas.1815237116

45. Yeh KY, Lo TS, Wu PM, Chang-Liao KS, Wang MJ, Wu MK. Magnetotransport studies of Fe-vacancy ordered $\mathrm{Fe}_{4+\delta} \mathrm{Se}_{5}$ nanowires. Proc Natl Acad Sci USA (2020) 116:1104. doi:10.1073/pnas.2000833117

46. Ma F, Ji W, Hu JP, Lu ZY, Xian T. First-principles calculations of the electronic structure of tetragonal $\alpha$-FeTe and $\alpha$-FeSe crystals: evidence for a bicollinear antiferromagnetic order. Phys Rev Lett (2009) 102:177003. doi:10.1103/ PhysRevLett.102.177003

47. Berlijn T, Hirschfeld PJ, Ku W. Effective doping and suppression of fermi surface reconstruction via Fe vacancy disorder in $\mathrm{K}_{\mathrm{x}} \mathrm{Fe}_{2-\mathrm{y}} \mathrm{Se}_{2}$. Phys Rev Lett (2012) 109:47003. doi:10.1103/PhysRevLett.109.147003

48. Zisese M, Blythe HJ. Magnetoresistance of magnetite. J Phys Condens Matter (2000) 13:12. doi:10.1088/0953-8984/12/1/302

49. Bohra M, Agarwal N, Sigh V. A short review on Verwey transition in nanostructured $\mathrm{Fe}_{3} \mathrm{O}_{4}$ materials. J Nanomater (2019) 1:18. doi:10.1155/ 2019/8457383

50. Kim T, Lim S, Hong J, Kwon SG, Okamoto J, Chen ZY, et al. Giant thermal hysteresis in Verwey transition of single domain $\mathrm{Fe}_{3} \mathrm{O}_{4}$ nanoparticles. Sci Rep (2018) 8:5092. doi:10.1038/s41598-018-23456-6

Conflict of Interest: Author PMW is employed by the company BitSmart LLC.

The remaining authors declare that the research was conducted in the absence of any commercial or financial relationships that could be construed as a potential conflict of interest.

Copyright ( $(2020$ Yeh, Chen, Lo, Wu, Wang, Chang-Liao and Wu. This is an openaccess article distributed under the terms of the Creative Commons Attribution License (CC BY). The use, distribution or reproduction in other forums is permitted, provided the original author(s) and the copyright owner(s) are credited and that the original publication in this journal is cited, in accordance with accepted academic practice. No use, distribution or reproduction is permitted which does not comply with these terms. 